\title{
Intussusception during Enteral Nutrition: A Case Report
}

\author{
TOSHIHIRO NOAKE, SHOGO YOSHIDA, HIROMASA FUJITA, \\ NOBUYA ISHIBASHI AND KAZUO SHIROUZU
}

\author{
Department of Surgery, Kurume University School of Medicine, \\ Kurume 830-0011, Japan
}

\begin{abstract}
Summary: We report a case of intussusception during enteral nutrition after esophagectomy. The case was a 60-year-old patient who underwent subtotal esophagectomy via the right thoracoabdominal approach, reconstructed with esophagogastrostomy. He underwent tube feeding through gastrostomy after surgery. The tip of the feeding tube was emplaced in the jejunum. He complained of intermittent abdominal pain and the drainage volume through the gastric decompression tube was increased on the 2 nd week from surgery. There was no abnormal finding on abdominal CT or on radiography of the digestive tract, and so a diagnosis of intussusception was made and laparotomy was performed. There was descending intussusception with the jejunum where the feeding tube was emplaced. Enterectomy was not necessary in this case. Adult intussusception is rare. The surgery is sometimes the cause for intussusception. Another cause is use of a long intestinal tube. Since enteral nutrition has priority for nutritional support after esophagectomy, it is important to make an early decision whether surgical intervention is required or not, using abdominal examination by $\mathrm{CT}$, ultrasound and contrast radiography, when bowel obstruction occurs during enteral nutrition through a feeding tube.
\end{abstract}

Key words intussusception, enteral nutrition, feeding tube

\section{INTRODUCTION}

Postoperative intussusception may occur due to various causes [1,2], and one of the reasons for intussusception is associated with the introduction of a tube in the digestive tract. Here report a case of intussusception during enteral nutrition through a feeding tube emplaced in the jejunum after esophagectomy.

\section{CASE REPORT}

\section{Patient: A 60-year-old man}

Primary disease: His primary disease was thoracic esophageal cancer. Surgical procedures were a subtotal esophagectomy and proximal gastrectomy via the right thoraco-abdominal approach, and the digestive tract was reconstructed with esophagogastrostomy, using a plastic stomach tube through a subcutaneous route. Gastrostomy was carried out for enteral feeding using a long 8Fr size tube (JMS Co., Hiroshima, Japan).

Postoperative course: Enteral nutrition (Twinline, Otsuka Pharmaceutical Co., Tokushima, Japan) was begun on the 5 th postoperative day with $400 \mathrm{kcal} /$ day at the rate of $100 \mathrm{ml} / \mathrm{hr}$. At 2 weeks after the surgery, intermittent abdominal pain occurred, and the volume of drainage from the gastric decompression tube was increased. A plain X-ray film of the abdomen showed no abnormal gas in the intestine. We performed abdominal computed tomography (CT) at 8 weeks after surgery to determine whether surely metastasis in a lymphnode caused a blocking intestinal passage. The CT revealed that there was a mass-like isodensity area accompanying a high-density area at the center, seemingly the nutrition tube (Fig. 1). Contrast radiography of the digestive tract using water soluble contrast medium (Gastrografin, Schering AG, Germany) showed nar- 


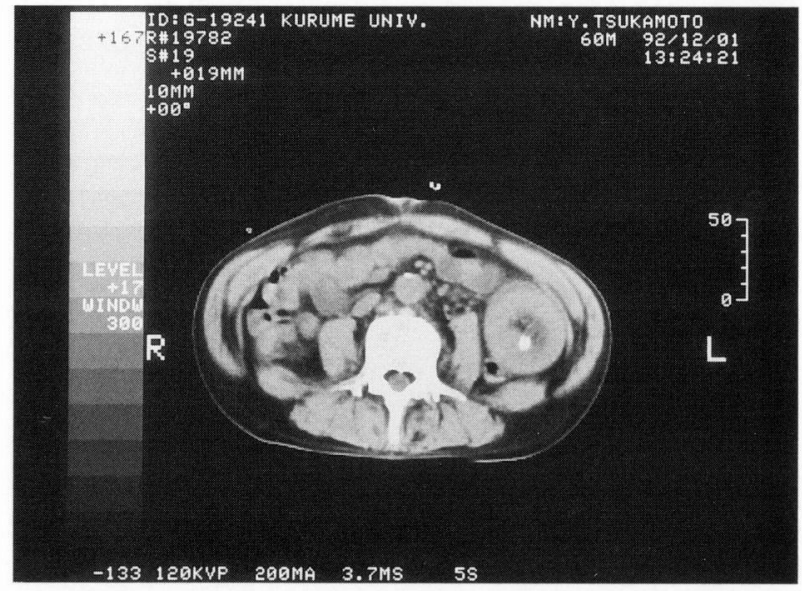

Fig. 1. Abdominal CT showed a mass-like isodensity area accompanied by a high-density area, seemingly the nutrition tube, in the lumen of the dilated intestinal tract.

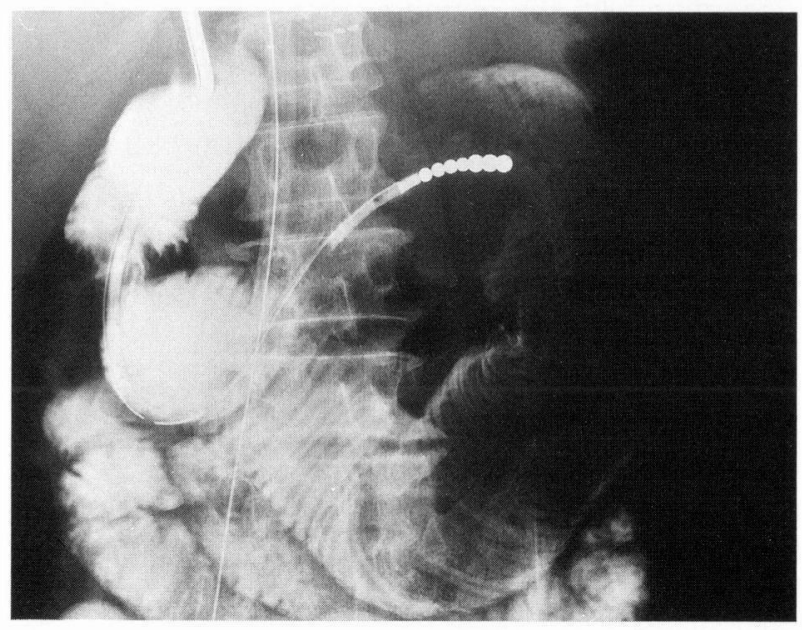

Fig. 2. On contrast radiography, injected water soluble contrast medium from the nutrition tube emplaced distal side from the stenosis showed a coiled spring sign.

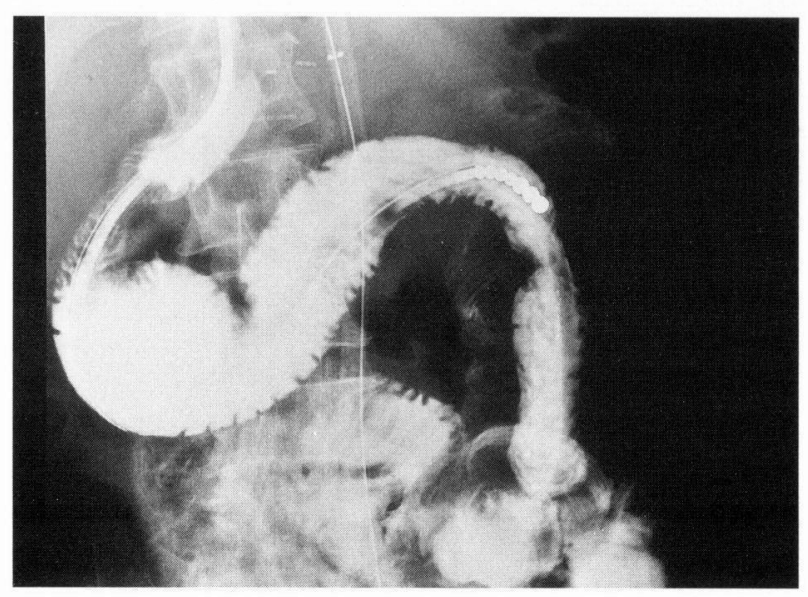

Fig. 3. On contrast radiography, water soluble contrast medium injected proximal to the stenosis via an ileus tube, showed stenosis along the nutrition tube. rowing in the jejunum along the nutrition tube (Fig. 1). Contrast radiography of the digestive tract using water soluble contrast medium (Gastrografin, Schering AG, Germany) showed narrowing in the jejunum along the nutrition tube with a coiled spring sign (Figs 2 and 3). These 2 studies suggested that the cause of the bowel obstruction was intussusception due to the enteral feeding tube. Therefore we performed reoperative surgery on the 57th day after the esophagectomy.

Findings during operation: In the jejunum at the site of the emplacement of the nutrition tube, when reduction was made manually, neither vascular disturbance nor organic narrowing was found. The operation was, therefore, completed without jejunectomy.

\section{DISCUSSION}

Postoperative intussusception occurs after various surgical procedures [3-7], in particular after gastric resection reconstructed by Billroth 2 method or Roux-en-Y anastomosis [5-7]. As to its etiology, Narita et al. [1] gave the factors shown in Table 1. In the light of these factors, the "Influence of double tract tube" and the "Infusion of drugs and/or nutritive solutions" were considered the many factors in our patient. Furthermore, "Spasm and abnormal peristalsis of the intestinal tract" may have been involved in our patient because it took 2 weeks for intestinal peristalsis to become normalized after the subtotal esophagectomy [8]. Hocking et al. [6] sus-

TABLE 1 .

Factors in the genesis of postoperative intussusception ${ }^{l}$

1. Mechanical factors

Elongated afferent loop

Lifting of afferent loop toward the gastric wall

Too large anastomotic wall

Drooping site of gastroenterostomy

Formation of postoperative adhesion

Narrowing accelerating antiperistalsis

Formation of cicatricial tissues

Influence of double tube

2. Function factors

Spasm and abnormal peristalsis of the intestinal tract

Stimulation caused by surgical manipulation

Postoperative inflammation

Abnormality of the autonomic nervous system

Infusion of drugs and/or nutritive solutions

Hypotension in the gastric wall hyperchylia

Elevation in abdominal pressure caused by vomiting, asthmatic attacks or others 
pected that abnormal motility played a role in the development of the intussusception using manometric evaluation. In our case, in particular, a nutrition tube was emplaced, and spasm in the intestinal tract at the site of emplacement and dilatation in the anal region of the intestinal tract caused by infusion of diet may have caused the intussusception.

In recent years, early postoperative enteral nutrition has been recommended, because enteral nutrition reduced the normal biological function [10]. The Nutritional Guidelines of the American Society for Parenteral and Enteral Nutrition (ASPEN) recommended the use of the gut, when the gut was functioning [11]. Enteral nutrition, using either gastrostomy or jejunostomy, is commonly employed for patient with esophagectomy.

It is important that the jejunum is sutured widely, and the small intestine is arranged smoothly so as not to bend, to avoid troubles in the jejunal tube. In addition, the following 3 items should be considered as preventing strategies for tube-related troubles: 1) a sufficient number of side holes are made in the tube to reduce the pressure and so stimuli are distributed not only to part of the intestinal tract, 2) the time of starting enteral nutrition is set after the discharge of gas being ascertained, and 3) enteral nutrition is started at a rate of $10-20 \mathrm{ml} / \mathrm{hr}$. After employing these strategies, we experienced no complications associated with the emplacement of the tube.

For diagnosis, after the occurrence of symptoms of postoperative ileus, then abdominal CT, ultrasonography, endoscopy and contrast radiography of the digestive tract are useful, in addition to plain radiography of the abdomen $[12,13]$. In our case, plain X-ray revealed no abnormality, because intussusception occurred in the upper jejunum and was proximal to the obstruction decompressed by the gastric decompression tube. CT scan or ultrasound study should have been performed earlier. Abdominal CT is especially useful for intussusception, and could have revealed the stage and etiology of intussusception objectively [14,15]. The ultrasonographic findings have been described as a target-like lesion, pseudo-kidney sign, or Hay-Fork sign [15-17], and ultrasonography is very useful because it can be performed at the bedside and can reveal the viability of the intussuscepted intestine. Radiography of the digestive tract is also useful, and "Coiled spring" sign is a characteristic radiographic finding of intussusception $[12,13]$. When contrast radiography is performed from the oral side, this finding can be detected in the case of ascending intussusception, but not, in the case of descending intussusception. Contrast radiography can also sometimes reveal narrowing in the intestine. In the present case, the "coiled spring" sign was shown on contrast radiography, since we performed this radiography from the anal side via the feeding tube.

We concluded that it is important to employ radiography on the digestive tract, ultrasonography on the abdomen and abdominal CT from the initial stage of bowel obstruction in order to avoid prolonged conservative therapy.

\section{REFERENCES}

1. Narita H, Ichino T, Koide H, Takeda Y, Watanabe S et al. Intussusception after laparotomy. Geka Chiryo (Surgical Therapy) 1983; 48:667-673. (in Japanese)

2. Sarr MG, Nagorney DM, and Mcilrath DC. Postoperative intussusception in the adult: a previously unrecognized entirely? Arch Surg 1981; 116:144-148.

3. Matley PJ. Postoperative intussusception in adults: a report of 5 cases. S Afr J Surg 1991; 29:30-32.

4. Kjellstrom BT, Gortz L, and Nilsson S. Adult idiopathic enteric intussusception in the postoperative intussusception in the postoperative period. Eur J Surg 1991; 157:359-360.

5. Denath FM, and Kweka EL. Retrograde intussusception of the bypassed duodenojejunal segment after Roux-en-Y gastrectomy: computed tomography findings. Can Assoc Radiol J 1991; 42:135-138.

6. Hocking MP, McCoy DM, Vogel SB, Kaude JV, and Sninsky CA. Antiperistaltic and isoperistaltic intussusception associated with abnormal motility after gastric bypass: a case report. Surgery 1991; 110:109-112.

7. Saxena R, Kochhar R, Nagi B, Mehta SK, and Gupta NM. Non-surgical treatment of jejunogastric intussusception. Surg Endosc 1988; 2:88-91.

8. Nagata K. Clinical study jejunal movement after radical operation for esophageal cancer. Nihon Shoukakigeka Gakkai Zasshi (Jpn J Gastroenterol Surg) 1986; 19:670679. (in Japanese)

9. Saito H, Trocki O, Alexander JW et al. The effect of route of nutrient state, catabolic hormone secretion and gut mucosal integrity after burn injury. JPEN 1987; 11:1.

10. Stein TP, Yoshida S, Schluter MD, Drews D, Assimon SA et al. Comparison of intravenous nutrients on gut mucosal protein synthesis. JPEN 1994; 18:447-452.

11. A.S.P.E.N. Board of Directors. Guidelines for the use of parenteral and enteral nutrition in adult and pediatric patient. JPEN 1993; 17 (Suppl):1-52SA.

12. Pollack CV, and Pender ES. Unusual case of intussusception. J Emerg Med 1991; 9:347-355.

13. Gordon RS, O’ Dell KB, Namon AJ, and Becker LB. Intussusception in the adult: a rare disease. J Emerg Med 1991; 9:337-342.

14. Bar-Ziv J, and Solomon A. Computed tomography in adult intussusception. Gastrointest Radiol 1991; 16:264- 
266.

15. Parienty RA, Lepreux JF, and Gruson B. Sonographic and CT features of ileocolic intussusception. AJR 1980; 136:608-610.

16. Seider R, and Darazsove B. Ultrasound diagnosis in intussusception of the bowel: a case report. S Afr Med J 1987; 72:151-152.

17. Alessi V, and Salerno G. The "Hay-Fork" sign in the ultrasonographic diagnosis of intussusception. Gastrointest Radiol 1985; 10:177-179. 